\title{
Submodular Functions, Matroids, and Certain Polyhedra $^{\star}$
}

\author{
Jack Edmonds \\ National Bureau of Standards, Washington, D.C., U.S.A.
}

\section{I}

The viewpoint of the subject of matroids, and related areas of lattice theory, has always been, in one way or another, abstraction of algebraic dependence or, equivalently, abstraction of the incidence relations in geometric representations of algebra. Often one of the main derived facts is that all bases have the same cardinality. (See Van der Waerden, Section 33.)

From the viewpoint of mathematical programming, the equal cardinality of all bases has special meaning - namely, that every basis is an optimumcardinality basis. We are thus prompted to study this simple property in the context of linear programming.

It turns out to be useful to regard "pure matroid theory", which is only incidentally related to the aspects of algebra which it abstracts, as the study of certain classes of convex polyhedra.

(1) A matroid $M=(E, F)$ can be defined as a finite set $E$ and a nonempty family $F$ of so-called independent subsets of $E$ such that

(a) Every subset of an independent set is independent, and

(b) For every $A \subseteq E$, every maximal independent subset of $A$, i.e., every basis of $A$, has the same cardinality, called the $\operatorname{rank}, r(A)$, of $A$ (with respect to $M)$.

(This definition is not standard. It is prompted by the present interest).

(2) Let $\mathbb{R}_{E}$ denote the space of real-valued vectors $x=\left[x_{j}\right], j \in E$.

Let $\mathbb{R}_{E}^{+}=\left\{x: 0 \leq x \in \mathbb{R}_{E}\right\}$.

(3) A polymatroid $P$ in the space $\mathbb{R}_{E}$ is a compact non-empty subset of $\mathbb{R}_{E}^{+}$ such that

(a) $0 \leq x^{0} \leq x^{1} \in P \Longrightarrow x^{0} \in P$.

(b) For every $a \in \mathbb{R}_{E}^{+}$, every maximal $x \in P$ such that $x \leq a$, i.e., every basis $x$ of $a$, has the same sum $\sum_{j \in E} x_{j}$, called the rank, $r(a)$, of $a$ (with respect to $P$ ).

* Synopsis for the Instructional Series of Lectures, "Polyhedral Combinatorics". 
Here maximal $x$ means that there is no $x^{\prime}>x$ having the properties of $x$.

(4) A polymatroid is called integral if (b) holds also when $a$ and $x$ are restricted to being integer-valued, i.e., for every integer-valued vector $a \in \mathbb{R}_{E}^{+}$, every maximal integer-valued $x$, such that $x \in P$ and $x \leq a$, has the same sum $\sum_{j \in E} x_{j}=r(a)$.

(Sometimes it may be convenient to regard an integral polymatroid as consisting only of its integer-valued members).

(5) Clearly, the $0-1$ valued vectors in an integral polymatroid are the "incidence vectors" of the sets $J \in F$ of a matroid $M=(E, F)$.

\section{II}

(6) Let $f$ be a real-valued function on a lattice $L$. Call it a $\beta_{0}$-function if

(a) $f(a) \geq 0$ for every $a \in K=L-\{\emptyset\}$;

(b) is non-decreasing: $a \leq b \Longrightarrow f(a) \leq f(b)$; and

(c) submodular:

$$
f(a \vee b)+f(a \wedge b) \leq f(a)+f(b)
$$

for every $a \in L$ and $b \in L$.

(d) Call it a $\beta$-function if, also, $f(\emptyset)=0$. In this case, $f$ is also subadditive, i.e., $f(a \vee b) \leq f(a)+f(b)$.

(We take the liberty of using the prefixes sub and super rather than "upper semi" and "lower semi". Semi refers to either. The term semi-modular is taken from lattice theory where it refers to a type of lattice on which there exists a semimodular function $f$ such that if $a$ is a maximal element less than element $b$ then $f(a)+1=f(b)$. See [1].)

(7) For any $x=\left[x_{j}\right] \in \mathbb{R}_{E}$, and any $A \subseteq E$, let $x(A)$ denote $\sum_{j \in A} x_{j}$.

(8) Theorem. Let $L$ be a family of subsets of $E$, containing $E$ and $\emptyset$, and closed under intersections, $A \cap B=A \wedge B$. Let $f$ be a $\beta_{0}$-function on $L$. Then the following polyhedron is a polymatroid:

$$
P(E, f)=\left\{x \in \mathbb{R}_{E}^{+}: x(A) \leq f(A) \text { for every } A \in L-\emptyset=K\right\} .
$$

Its rank function $r$ is, for any $a=\left[a_{j}\right] \in \mathbb{R}_{E}^{+}$,

$$
r(a)=\min \left(\sum_{j \in E} a_{j} z_{j}+\sum_{A \in K} f(A) y_{A}\right)
$$

where the $z_{j}$ 's and $y_{A}$ 's are 0 's and 1's such that for every $j \in E$, 


$$
z_{j}+\sum_{j \in A \in K} y_{A} \geq 1
$$

Where $f(\emptyset) \geq 0$, only one non-zero $y_{A}$ is needed.

Where $f$ is integer-valued, $P(E, f)$ is an integral polymatroid.

(9) Theorem. A function $f$ of all sets $A \subseteq E$ is itself the rank function of a matroid $M=(E, F)$ iff it is an integral $\beta$-function such that $f(\{j\})=1$ or 0 for every $j \in E$. Such an $f$ determines $M$ by:

$$
J \in F \Longleftrightarrow J \subseteq E \text { and }|J|=f(J) .
$$

(10) For any $a=\left[a_{j}\right] \in \mathbb{R}_{E}^{+}$and $b=\left[b_{j}\right] \in \mathbb{R}_{E}^{+}$, let $a \vee b=\left[u_{j}\right] \in \mathbb{R}_{E}^{+}$and $a \wedge b=\left[v_{j}\right] \in \mathbb{R}_{E}^{+}$, where

$$
u_{j}=\max \left(a_{j}, b_{j}\right) \quad \text { and } \quad v_{j}=\min \left(a_{j}, b_{j}\right) .
$$

(11) Theorem. The rank function $r(a), a \in \mathbb{R}_{E}^{+}$, for any polymatroid $P \subset$ $\mathbb{R}_{E}^{+}$, is a $\beta$-function on $\mathbb{R}_{E}^{+}$relative to the above $\vee$ and $\wedge$.

(12) For any $x=\left[x_{j}\right] \in \mathbb{R}_{E}^{+}$and any $A \subseteq E$, let $x / A=\left[(x / A)_{j}\right] \in \mathbb{R}_{E}^{+}$denote the vector such that $(x / A)_{j}=x_{j}$ for $j \in A$, and $(x / A)_{j}=0$ for $j \notin A$.

(13) Given a polymatroid $P \subset \mathbb{R}_{E}^{+}$, let $\alpha \in \mathbb{R}_{E}^{+}$be an integer-valued vector such that $x<\alpha$ for every $x \in P$. Where $r$ is the rank function of $P$, let $f_{P}(A)=r(\alpha / A)$ for every $A \subseteq E$.

Let $L_{E}=\{A: A \subseteq E\}$. Clearly, by (11), $f_{P}$ is a $\beta$-function on $L_{E}$. Furthermore, if $P$ is integral, then $f$ is integral.

Theorem. For any polymatroid $P \subset \mathbb{R}_{E}^{+}$,

$$
P=P\left(E, f_{P}\right)
$$

Thus, all polymatroids $P \in \mathbb{R}_{E}^{+}$are polyhedra, and they correspond to certain $\beta$-functions on $L_{E}$.

Theorem 8 provides a useful way of constructing matroids which is quite different from the usual algebraic constructions.

(15) For any given integral $\beta_{0}$-function $f$ as in (8), let a set $J \subseteq E$ be a member of $F$ iff for every $A \in K=L-\{\emptyset\},|J \cap A| \leq f(A)$. In particular, where $L=L_{E}$, let a set $J \subseteq E$ be a member of $F$ when for every $\emptyset \neq A \subseteq J,|A| \leq f(A)$. Then (8) implies that $M=(E, F)$ is a matroid, and gives a formula for its rank function in terms of $f$. (This generalizes a construction given by Dilworth [1]). 
In this section, $K$ will denote $L_{E}-\{\emptyset\}=\{A: \emptyset \neq A \subseteq E)$.

(16) Given any $c=\left\{c_{j}\right\} \in \mathbb{R}_{E}$, and given a $\beta$-function $f$ on $L_{E}$, we show how to solve the linear program:

$$
\operatorname{maximize} c \cdot x=\sum_{j \in E} c_{j} x_{j} \text { over } x \in P(E, f) .
$$

(17) Let $j(1), j(2), \ldots$ be an ordering of $E$ such that

$$
c_{j(1)} \geq c_{j(2)} \geq \cdots c_{j(k)}>0 \geq c_{j(k+1)} \geq \cdots
$$

(18) For each integer $i, 1 \leq i \leq k$, let

$$
A_{i}=\{j(1), j(2), \ldots, j(i)\} .
$$

(19) Theorem. (The Greedy Algorithm). $\quad c \cdot x$ is maximized over $x \in P(E, f)$ by the following vector $x^{0}$ :

$$
\begin{array}{ll}
x_{j(1)}^{0}=f\left(A_{1}\right) ; & \\
x_{j(i)}^{0}=f\left(A_{i}\right)-f\left(A_{i-1}\right) & \text { for } 2 \leq i \leq k ; \\
x_{j(i)}^{0}=0 & \text { for } k<i \leq|E| .
\end{array}
$$

(There is a well-known non-polyhedral version of this for graphs, given by Kruskal [9]. A related theorem for matroids is given by Rado [15]).

The dual l.p. is to minimize

$$
f \cdot y=\sum_{A \in K} f(A) y(A) \text { where }
$$

(20) $y(A) \geq 0$; and for every $j \in E, \sum_{j \in A} y(A) \geq c_{j}$.

(21) Theorem. An optimum solution, $y^{0}=\left[y^{0}(A)\right], A \in K$, to the dual l.p. is

$$
\begin{aligned}
y^{0}\left(A_{i}\right) & =c_{j(i)}-c_{j(i+1)} & & \text { for } 1 \leq i \leq k-1 \\
y^{0}\left(A_{k}\right) & =c_{j(k)} ; \quad \text { and } \quad y^{0}(A)=0 & & \text { for all other } A \in K .
\end{aligned}
$$

(22) Theorem. Corollary to (19). The vertices of the polyhedron $P(E, f)$ are precisely the vectors of the form $x^{0}$ in (19) for some sequence $j(1), j(2), \ldots, j(k)$. 
(23) Where $f$ is the rank function of a matroid $M=(E, F),(9)$ and (22) imply that the vertices of $P(E, f)$ are precisely the incidence vectors of the members of $F$, i.e., the independent sets of $M$. Such a $P(E, f)$ is called a matroid polyhedron.

(24) Let $f$ be a $\beta$-function on $L_{E}$. A set $A \in L_{E}$ is called $f$-closed or an $f$-flat, when, for any $C \in L_{E}$ which properly contains $A, f(A)<f(C)$.

(25) Theorem. If $A$ and $B$ are $f$-closed then $A \cap B$ is $f$-closed.

(In particular, for the $f$ of $(9)$, the $f$-flats form a "geometric" or "matroid" lattice.)

Proof: Suppose that $C$ properly contains $A \cap B$. Then either $C \nsubseteq A$ or $C \nsubseteq B$. Since $f$ is non-decreasing we have $f(A \cap B) \leq f(A \cap C)$ and $f(A \cap B) \leq f(B \cap C)$. Thus, since $f$ is submodular, we have either

$$
\begin{aligned}
& 0<f(A \cup C)-f(A) \leq f(C)-f(A \cap C) \leq f(C)-f(A \cap B), \quad \text { or } \\
& 0<f(B \cup C)-f(B) \leq f(C)-f(B \cap C) \leq f(C)-f(A \cap B) .
\end{aligned}
$$

(26) A set $A \in K$ is called $f$-separable when

$$
f(A)=f\left(A_{1}\right)+f\left(A_{2}\right)
$$

for some partition of $A$ into non-empty subsets $A_{1}$ and $A_{2}$. Otherwise $A$ is called $f$-inseparable.

(27) Theorem. Any $A \in K$ partitions in only one way into a family of $f$ inseparable sets $A_{i}$ such that $f(A)=\sum f\left(A_{i}\right)$. The $A_{i}$ 's are called the $f$-blocks of $A$.

If a polyhedron $P \subset \mathbb{R}_{E}$ has dimension equal to $|E|$ then there is a unique minimal system of linear inequalities having $P$ as its set of solutions. These inequalities are called the faces of $P$.

It is obvious that a polymatroid $P \subset \mathbb{R}_{E}^{+}$has dimension $|E|$ if and only if, where $f$ is the $\beta$-function which determines it, and set $\emptyset$ is $f$-closed. It is obvious that inequality $x(A) \leq f(A), A \in K$, is a face of $P(E, f)$ only if $A$ is $f$-closed and $f$-inseparable.

(28) Theorem. Where $f$ is a $\beta$-function on $L_{E}$ such that the empty set is $f$-closed, the faces of polymatroid $P(E, f)$ are: $x_{j} \geq 0$ for every $j \in E$; and $x(A) \leq f(A)$ for every $A \in K$ which is $f$-closed and $f$-inseparable.

\section{IV}

(29) Let each $V_{p}, p=1$ and 2 , be a family of disjoint subsets of $H$. Where $\left[a_{i j}\right]$, $i \in E, j \in E$, is the $0-1$ incidence matrix of $V_{1} \cup V_{2}=H$, the following l.p. is known as the Hitchcock problem. 
(30) Maximize $c \cdot x=\sum_{j \in E} c_{j} x_{j}$, where

(31) $x_{j} \geq 0$ for every $j \in E$, and $\sum_{j \in E} a_{i j} x_{j} \leq b_{i}$ for every $i \in H$.

The dual l.p. is

(32) Minimize $b \cdot y=\sum b_{i} y_{i}$, where

(33) $y_{i} \geq 0$ for every $i \in H$, and $\sum_{i \in H} a_{i j} y_{i} \geq c_{j}$ for every $j \in E$.

Denote the polyhedron of solutions of a system $Q$ by $P[Q]$.

The following properties of the Hitchcock problem are important in its combinatorial use.

(34) Theorem. (a) Where the $b_{i}$ 's are integers, the vertices of $P[(31)]$ are integer-valued. $(b)$ Where the $c_{j}$ 's are integers, the vertices of $P[(33)]$ are integervalued.

Theorem (34a) generalizes to the following.

(35) Theorem. For any two integral polymatroids $P_{1}$ and $P_{2}$ in $\mathbb{R}_{E}^{+}$, the vertices of $P_{1} \cap P_{2}$ are integer-valued.

The following technique for proving theorems like (34) is due to Alan Hoffman [7.

(36) Theorem. The matrix $\left[a_{i j}\right]$ of the Hitchcock problem is totally unimodular - that is, the determinant of every square submatrix has value 0,1 , or -1 .

(37) Theorem. Theorem (34) holds whenever $\left[a_{i j}\right]$ is totally unimodular.

(38) Let each $V_{p}, p=1$ and 2, be a family of subsets of $E$ such that any two members of $P$ are either disjoint or else one is a subset of the other.

(39) Theorem. The incidence matrix of the $V_{1} \cup V_{2}$ of (38) is totally unimodular.

Property (29) is a special case of (38). Property (38) is a special case of the following.

(40) Let each $V_{p}, p=1$ and 2, be a family of subsets of $E$ such that for any $R \in V_{p}$ and $S \in V_{p}$ either $R \cap S=\emptyset$ or $R \cap S \in V_{p}$.

The incidence matrix of the $V_{1} \cup V_{2}$ of (40) is generally not totally unimodular. However,

(41) Theorem. From the incidence matrix of each $V_{p}$ of (40), once can obtain, by subtracting certain rows from others, the incidence matrix of a family of mutually disjoint subsets of E. Thus, in the same way, one can obtain from the incidence matrix of the $V_{1} \cup V_{2}$ of (40), a matrix of the Hitchcock type.

(42) Theorem. For any polymatroid $P(E, f)$ and any $x \in P(E, f)$, if $x(A)=$ $f(A)$ and $x(B)=f(B)$ then either $A \cap B=\emptyset$ or $x(A \cap B)=f(A \cap B)$. 
Theorems (42), (41), and (34a) imply (35).

(43) Assuming that each $V_{p}$ of (38) contains the set $E, L_{p}=V_{p} \cup\{\emptyset\}$ is a particularly simple lattice. For any non-negative non-decreasing function $f(i)=$ $b_{i}, i \in V_{p}$, let $f(\emptyset)=-f(E)$. Then $f$ is a $\beta_{0}$-function on $L_{p}$.

(44) The only integer vectors in a matroid polyhedron $P$ are the vectors of the independent sets of the matroid, and these vectors are all vertices of $P$. Thus, (35) implies:

(45) Theorem. Where $P_{1}$ and $P_{2}$ are the polyhedra of any two matroids $M_{1}$ and $M_{2}$ on $E$, the vertices of $P_{1} \cap P_{2}$ are precisely the vectors which are vertices of both $P_{1}$ and $P_{2}$ - namely, the incidence vectors of sets which are independent in both $M_{1}$ and $M_{2}$.

Where $P_{1}, P_{2}$, and $P_{3}$ are the polyhedra of three matroids on $E$, polyhedron $P_{1} \cap P_{2} \cap P_{3}$ generally has many vertices besides those which are vertices of $P_{1}, P_{2}$, and $P_{3}$.

Let $c=\left[c_{j}\right], j \in E$, be any numerical weighting of the elements of $E$. In view of (45), the problem:

(46) Find a set $J$, independent in both $M_{1}$ and $M_{2}$, that has maximum weightsum, $\sum_{j \in J} c_{j}$, is equivalent to the l.p. problem:

(47) Find a vertex $x$ of $P_{1} \cap P_{2}$ that maximizes $c \cdot x$.

(48) Assuming there is a good algorithm for recognizing whether of not a set $J \subseteq E$ is independent in $M_{1}$ or in $M_{2}$, there is a good algorithm for problem (46). This seems remarkable in view of the apparent complexity of matroid polyhedra in other respects. For example, a good algorithm is not known for the problem:

(49) Given a matroid $M_{1}=\left(E, F_{1}\right)$ and given an element $e \in E$, minimize $|D|$, $D \subseteq E$, where $e \in D \notin F_{1}$;

Or the problems:

(50) Given three matroids $M_{1}, M_{2}$, and $M_{3}$, on $E$, and given an objective vector $c \in \mathbb{R}_{E}$, maximize $c \cdot x$ where $x \in P_{1} \cap P_{2} \cap P_{3}$.

Or maximize $\sum_{j \in J} c_{j}$ where $J \in F_{1} \cap F_{2} \cap F_{3}$.

\section{V}

Where $f_{1}$ and $f_{2}$ are $\beta$-functions on $L_{E}$, the dual of the l.p.:

(51) Maximize $c \cdot x=\sum_{j \in E} c_{j} x_{j}$, where

(52) For every $j \in E, x_{j} \geq 0$; and for every $A \in K, x(A) \leq f_{1}(A)$ and $x(A) \leq f_{2}(A)$; is the l.p.: 


$$
\text { Minimize } f \cdot y=\sum_{A \in K}\left[f_{1}(A) y_{1}(A)+f_{2}(A) y_{2}(A)\right]
$$

where

(54) For every $A \in K, y_{1}(A) \geq 0$ and $y_{2}(A) \geq 0$; and for every $j \in E$,

$$
\sum_{j \in A \in K}\left[y_{1}(A)+y_{2}(A)\right] \geq c_{j} .
$$

Combining systems (52) and (54) we get,

$$
\begin{aligned}
& \sum_{j \in E} x_{j}\left(\sum_{j \in A \in K}\left[y_{1}(A)+y_{2}(A)\right]-c_{j}\right) \\
& +\sum_{A \in K} y_{1}(A)\left[f_{1}(A)-\sum_{j \in A} x_{j}\right] \\
& +\sum_{A \in K} y_{2}(A)\left[f_{2}(A)-\sum_{j \in A} x_{j}\right] \geq 0 .
\end{aligned}
$$

Expanding and cancelling we get

$$
c \cdot x \leq f \cdot y
$$

for any $x$ satisfying (52) and any $y=\left(y_{1}, y_{2}\right)$ satisfying (54).

(57) Equality holds in (56) if and only if equality holds in (55).

The l.p. duality theorem says that

(58) If there is an $x^{0}$, a vertex of $P[(52)]$, which maximizes $c \cdot x$, then there is a $y^{0}=\left(y_{1}^{0}, y_{2}^{0}\right)$, a vertex of $P[(54)]$, such that

$$
c \cdot x^{0}=f \cdot y^{0}
$$

and hence such that $y^{0}$ minimizes $f \cdot y$.

For the present problem obviously there is such an $x^{0}$.

The vertices of (54) are not generally all integer-valued when the $c_{j}$ 's are. However,

(60) Theorem. If the $c_{j}$ 's are all integers, then, regardless of whether $f_{1}$ and $f_{2}$ are integral, there is an integer-valued solution $y^{4}=\left(y_{1}^{4}, y_{2}^{4}\right)$ of (54) which minimizes $f \cdot y$.

Let $y^{3}=\left(y_{1}^{3}, y_{2}^{3}\right)$ be any solution of (54) which minimizes $f \cdot y$.

(61) For every $j \in E$, and $p=1,2$ let $c_{j}^{p}=\sum_{j \in A \in K} y_{p}^{3}$

For each $p=1,2$ consider the problem, 
(62) Minimize $f_{p} \cdot y_{p}=\sum_{A \in K} f_{p}(A) y_{p}(A)$ where

(63) for every $A \in K, y_{p}(A) \geq 0$; and for every $j \in E$,

$$
\sum_{j \in A \in K} y_{p}(A) \geq c_{j}^{p} .
$$

(64) By (21) for each $p$, there is an optimum solution, say $y_{p}^{4}$, to (62) having the following form:

(65) The sets $A \in K$, such that $y_{p}^{4}(A)>0$, form a nested sequence,

$$
A_{1} \subset A_{2} \subset A_{3} \subset \ldots
$$

Since $y_{p}^{3}$ is a solution of (63), we have $f_{p} y_{p}^{4} \leq f_{p} y_{p}^{3}$, for each $p$, and thus $f \cdot y^{4} \leq f \cdot y^{3}$. Since $c_{j}^{1}+c_{j}^{2} \geq c_{j}$ for every $j \in E, y^{4}$ is a solution of (54), and hence $y^{4}$ is an optimum solution of (54). Thus, we have that

(66) Theorem. There exists a solution $y^{4}$ of (54) which minimizes $f \cdot y$ and which has property (65) for each $p=1,2$.

The problem, minimize $f \cdot y$ subject to $(54)$ and also subject to $y_{p}(A)=0$ for every $y_{p}^{4}(A)=0$, has the form $[(32),(33)]$ where $\left[a_{i j}\right]$ is the incidence matrix of a $V_{1} \cup V_{2}$ as in (38). Thus, by (39) and (37), we have:

(67) Theorem. If the $c_{j}$ 's are all integers then the $y^{4}$ of (66) can be taken to be integer-valued.

In particular this proves (60).

An immediate consequence of (35), (60), and the l.p. duality theorem is

(68) Theorem. $\max c \cdot x=\min f \cdot y$ where $x \in P[(52)]$ and $y \in P[(54)]$.

If $f$ is integral, $x$ can be integral.

If $c$ is integral, $y$ can be integral.

In particular, where $f_{1}$ and $f_{2}$ are the rank functions, $r_{1}$ and $r_{2}$, of any two matroids, $M_{1}=\left(E, F_{1}\right)$ and $M_{2}=\left(E, F_{2}\right)$, and where every $c_{j}=1$, (68) implies:

(69) Theorem. $\max |J|=\min \left[r_{1}(S)+r_{2}(E-S)\right]$, where $J \in F_{1} \cap F_{2}$, and where $S \subseteq E$.

(A related result is given by Tutte [16]).

\section{VI}

(70) Theorem. For each $i \in E^{\prime}$, let $Q_{i}$ be a subset of $E$. For each $A^{\prime} \subseteq E^{\prime}$, let $u\left(A^{\prime}\right)=\bigcup_{i \in A^{\prime}} Q_{i}$. Let $f$ be any integral $\beta$-function on $L_{E}$. 

$\left.E^{\prime}\right\}$.

Then $f^{\prime}\left(A^{\prime}\right)=f\left(u\left(A^{\prime}\right)\right)$ is an integral $\beta$-function on $L_{E^{\prime}}=\left\{A^{\prime}: A^{\prime} \subseteq\right.$

(71) This follows from the relations

$$
\begin{aligned}
& u\left(A^{\prime} \cup B^{\prime}\right)=u\left(A^{\prime}\right) \cup u\left(B^{\prime}\right) \quad \text { and } \\
& u\left(A^{\prime} \cap B^{\prime}\right) \subseteq u\left(A^{\prime}\right) \cap u\left(B^{\prime}\right) .
\end{aligned}
$$

(72) Applying (15) to $f^{\prime}$ yields a matroid on $E^{\prime}$.

(73) In particular, taking $f$ to mean cardinality, if we let $J^{\prime} \subseteq E^{\prime}$ be a member of $F^{\prime}$ iff $\left|A^{\prime}\right| \leq\left|u\left(A^{\prime}\right)\right|$ for every $A^{\prime} \subseteq J^{\prime}$, then $M^{\prime}=\left(E^{\prime}, F^{\prime}\right)$ is a matroid.

(74) Hall's SDR theorem says that: $\left|A^{\prime}\right| \leq\left|u\left(A^{\prime}\right)\right|$ for every $A^{\prime} \subseteq J^{\prime}$ iff the family $\left\{Q_{j}\right\}, i \in J^{\prime}$, has a system of distinct representatives, i.e., a transversal. A transversal of a family $\left\{Q_{i}\right\}, i \in J^{\prime}$ is a set $\left\{j_{i}\right\}, i \in J^{\prime}$, of distinct elements such that $j_{i} \in Q_{i}$. Thus,

(75) Theorem. For any finite family $\left\{Q_{i}\right\}, i \in E^{\prime}$, of subsets of $E$, the sets $J^{\prime} \subseteq E^{\prime}$ such that $\left\{Q_{i}\right\}, i \in J^{\prime}$, has a transversal are the independent sets of a matroid on $E^{\prime}$ (called a transversal matroid).

There are a number of interesting ways to derive (75). Some others are in [2], 3], [5], and [12. The present derivation is the way (75) was first obtained and communicated.

The following is the same result with the roles of elements and sets interchanged.

(76) Let $J \in F_{0}$ iff, for some $J^{\prime} \subseteq E^{\prime}, J$ is a transversal of $\left\{Q_{i}\right\}, i \in J^{\prime}$. That is, let $J \in F_{0}$ iff $J$ is a partial transversal of $\left\{Q_{i}\right\}, i \in E^{\prime}$. Then $M_{0}=\left(E, F_{0}\right)$ is a matroid.

(77) Thus, where $P_{0}$ is the polyhedron of $M_{0}$ and where $P$ is the polyhedron of any other matroid, $M=(E, F)$, on $E$, the vertices of $P_{0} \cap P$ are the incidence vectors of the $M$-independent partial transversals of $\left\{Q_{i}\right\}, i \in E^{\prime}$.

By (8), the rank function $r_{0}$ of $M_{0}$ is, for each $A \subseteq E$,

$r_{0}(A)=\min \left[\left|A_{0}\right|+\left|\left\{i:\left(A-A_{0}\right) \cap Q_{i} \neq \emptyset\right\}\right|\right]$ where $A_{0} \subseteq A$.

Combining (69) and (78), we get

$$
\begin{aligned}
& \max |J|=\min \left[r\left(A_{1}\right)+\left|A_{0}\right|+\left|E^{\prime}\right|-\left|\left\{i: Q_{i} \subseteq A_{1} \cup A_{0}\right\}\right|\right] \\
= & \min \left[r\left(u\left(A^{\prime}\right)\right)+\left|E^{\prime}\right|-\left|A^{\prime}\right|\right], \text { where } J \in F_{0} \cap F, A_{0} \cup A_{1} \subseteq E, A_{0} \cap A_{1}=\emptyset,
\end{aligned}
$$
and $A^{\prime} \subseteq E^{\prime}$.

In particular, (79)implies the following theorem of Rado 14, given in 1942.

(80) For any matroid $M$ on $E$, a family $\left\{Q_{i}\right\}, i \in E^{\prime}$, of subsets of $E$, has a transversal which is independent in $M$ iff $\left|A^{\prime}\right| \leq r\left(u\left(A^{\prime}\right)\right)$ for every $A^{\prime} \subseteq E^{\prime}$. 
Taking the $f$ of (70) to be $r,(70),(15)$, and (80) imply:

(81) Theorem. For any matroid $M$ on $E$, and any family $\left\{Q_{i}\right\}, i \in E^{\prime}$, of subsets of $E$, the sets $J^{\prime} \subseteq E^{\prime}$ such that $\left\{Q_{i}\right\}, i \in J^{\prime}$, has an $M$-independent transversal are the independent sets of a matroid on $E^{\prime}$.

(82) A bipartite graph $G$ consists of two disjoint finite sets, $V_{1}$ and $V_{2}$, of nodes and a finite set $E(G)$ of edges such that each member of $E(G)$ meets one node in $V_{1}$ and one node in $V_{2}$.

The following theorem of König is a prototype of (69).

(83) Theorem. For any bipartite graph $G, \max |J|, J \subseteq E(G)$, such that

(a) no two members of $J$ meet the same node in $V_{1}$, and

(b) no two members of $J$ meet the same node in $V_{2}$,

equals $\min \left(\left|T_{1}\right|+\left|T_{2}\right|\right), T_{1} \subseteq V_{1}$, and $T_{2} \subseteq V_{2}$, such that every member of $E(G)$ meets a node in $T_{1}$ or a node in $T_{2}$.

(84) To get the Hall theorem, (74), from (83), let $V_{1}$ be the $E^{\prime}$ of (70), let $V_{2}$ be the $E$ of (70), and let there be an edge in $E(G)$ which meets $i \in V_{1}$ and $j \in V_{2}$ iff $j \in Q_{i}$.

Clearly, if the family $\left\{Q_{i}\right\}, i \in E^{\prime}$, has no transversal then, in (83), $\max |J|<\left|V_{1}\right|$. If the latter holds, then by (83), the $T_{1}$ of $\min \left(\left|T_{1}\right|+\left|T_{2}\right|\right)$, in (83), is such that

$$
\left|V_{1}-T_{1}\right|>\left|u\left(V_{1}-T_{1}\right)\right|
$$

(85) For the König-theorem instance, (83) of (69), the matroids $M_{1}=\left(E, F_{1}\right)$ and $M_{2}=\left(E, F_{2}\right)$ are particularly simple: Let $E=E(G)$. For $p=1$ and $p=2$, let $J \subseteq E(G)$ be a member of $F_{p}$ iff no two members of $J$ meet the same node in $V_{p}$.

(86) Where $P_{1}$ and $P_{2}$ are the polyhedra of these two matroids, finding a vertex $x$ of $P_{1} \cap P_{2}$ which maximizes $c \cdot x$ is essentially the optimal assignment problem. That is, the Hitchcock problem where every $b_{i}=1$.

(87) Clearly, the inequality $x(A) \leq r_{p}(A)$ is a face of $P_{p}$, that is, $A$ is $r_{p}$-closed and $r_{p}$-inseparable, iff, for some node $v \in V_{p}, A$ is the set of edges which meet $v$.

\section{VII}

(88) Let $\left\{M_{i}\right\}, i \in I$, be a family of matroids, $M_{i}=\left(E, F_{i}\right)$, having rank functions $r_{i}$. Let $J \subseteq E$ be a member of $F$ iff:

(89) $|A| \leq \sum_{i} r_{i}(A)$ for every $A \subseteq J$. 
Since $f(A)=\sum_{i} r_{i}(A)$ is a $\beta$-function on $L_{E}$,

(90) Theorem. The $M=(E, F)$ of (88) is a matroid, called the sum of the matroids $M_{i}$.

In [5], and in [2], it is shown that

(91) Theorem. $J \subseteq E$ satisfies (89) iff $J$ can be partitioned into sets $J_{i}$ such that $J_{i} \in F_{i}$.

(92) An algorithm, MPAR, is given there for either finding such a partition of $J$ or else finding an $A \subseteq J$ which violates (89). That is, for recognizing whether or not $J \in F$.

(93) The algorithm is a good one, assuming:

(94) that a good algorithm is available for recognizing, for any $K \subseteq E$ and for each $i \in I$, whether or not $K \in F_{i}$.

(95) The definition of a matroid $M=(E, F)$ is essentially that, modulo the ease of recognizing, for any $J \subseteq E$, whether or not $J \in F$, one has what is perhaps the easiest imaginable algorithm for finding, in any $A \subseteq E$, a maximum cardinality subset $J$ of A such that $J \in F$.

(96) In particular, by virtue of (90), assuming (94), MPAR provides a good algorithm for finding a maximum cardinality set $J \subseteq E$ which is partitionable into sets $J_{i} \in F_{i}$.

(97) Assuming (94), MPAR combined with (19) is a good algorithm for, given numbers $c_{j}, j \in E$, finding a set $J$ which is partitionable into sets $J_{i} \in F_{i}$ and such that $\sum_{j \in J} c_{j}$ is maximum.

Where $r$ is the rank function of matroid $M=(E, F)$, let

$r^{*}(A)=|A|+r(E-A)-r(E)$ for every $A \subseteq E$.

Substituting $r(E)=|E|-r^{*}(E)$, and $A$ for $E-A$, in (98), yields

$$
r(A)=|A|+r^{*}(E-A)-r^{*}(E) .
$$

(100) It is easy to verify that $r^{*}$ is the rank function of a matroid $M^{*}=\left(E, F^{*}\right)$, e.g., that $r^{*}$ satisfies (9). $M^{*}$ is called the dual of $M$. By (99), $M^{* *}=M$.

(101) By (98), $|J|=r^{*}(J)$ iff $r(E-J)=r(E)$. Therefore, $J \in F^{*}$ iff $E-J$ contains an $M$-basis of $E$, i.e., a basis of $M$. Thus, it can be determined whether or not $J \in F^{*}$ by obtaining an $M$-basis of $E-J$ and observing whether or not its cardinality equals $r(E)$.

Where $r$ is the rank function of a matroid $M=(E, F)$, and where $n$ is a non-negative integer, let

(102) $r^{(n)}(A)=\min [n, r(A)]$ for every $A \subseteq E$. 
(103) Clearly, $r^{(n)}$ is the rank function of a matroid $M^{(n)}=\left(E, F^{(n)}\right)$, called the $n$-truncation of $M$, such that $J \in F^{(n)}$ iff $J \in F$ and $|J| \leq n$.

(104) For matroids $M_{1}=\left(E, F_{1}\right)$ and $M_{2}=\left(E, F_{2}\right)$, and any integer $n \leq r_{2}(E)$, by (103) and (101), there is a set $J \in F_{1} \cap F_{2}$ such that $|J|=n$ iff $E$ can be partitioned into a set $J_{1} \in F_{1}$ and a set $J_{2} \in F_{2}^{(n)^{*}}$. Theorem (91) says this is possible iff $|A| \leq r_{1}(A)+r_{2}^{(n)^{*}}(n)(A)$ for every $A \subseteq E$. Using (102) and (98), this implies (69).

(105) Using $M P A R$, a maximum cardinality $J \in F_{1} \cap F_{2}$ can be found as follows: Find a maximum cardinality set $H=J_{1} \cup J_{2}$ such that $J_{1} \in F_{1}$ and $J_{2} \in F_{2}^{*}$. Extend $J_{2}$ to $B$, an $M_{2}^{*}$-basis of $H$. Clearly, $B$ is an $M_{2}^{*}$-basis of $E$, and so $H-B \in F_{1} \cap F_{2}$. It is easy to verify that $|H-B|=\max |J|, J \in F_{1} \cap F_{2}$.

(106) It is more practical to go in the other direction, obtaining for a given family of matroids $M_{i}=\left(E, F_{i}\right), i \in I$, an "optimum" family of mutually disjoint sets $J_{i} \in F_{i}$, by using the "matroid intersection algorithm" of (48) on the following two matroids $M_{1}=\left(E_{I}, F_{1}\right)$ and $M_{2}=\left(E_{I}, F_{2}\right)$. Let $E_{I}$ consist of all pairs $(j, i)$, $j \in E$ and $i \in I$. There is a 1-1 correspondence between sets $J \in E_{I}$ and families $\left\{J_{i}\right\}, i \in I$, of sets $J_{i} \subseteq E$, where $J$ corresponds to the family $\left\{J_{i}\right\}$ such that $j \in J_{i} \Longleftrightarrow(j, i) \in J$. Let $M_{1}=\left(E_{I}, F_{1}\right)$ be the matroid such that $J \subseteq E_{I}$ is a member of $F_{1}$ iff the corresponding sets $J_{i}$ are mutually disjoint - that is, if and only if the $j$ 's of the members of $J$ are distinct. Let $M_{2}=\left(E_{I}, F_{2}\right)$ be the matroid such that $J \subseteq E_{I}$ is a member of $F_{2}$ iff the corresponding sets $J_{i}$ are such that $J_{i} \in F_{i}$. way [13.)

(Nash-Williams has developed the present subject in another interesting

\section{VIII}

(107) If $f(a)$ is a $\beta$-function on $L$ and $k$ is a not-too-large constant, then $f(a)-k$ is a $\beta_{0}$-function on $L$. It is useful to apply (15) to, non- $\beta, \beta_{0}$-functions.

(108) For example, let $G$ be a graph having edge-set $E=E(G)$ and node-set $V=V(G)$. For each $j \in E$, let $Q_{j}$ be the set of nodes which $j$ meets. For every $A \subseteq E$, let $f(A)=|u(A)|-1$. Then, by (70), $f(A)$ is a $\beta_{0}$-function on $L_{E}$.

(109) Applying (15) to this $f$ yields a matroid, $M(G)=(E, F(G))$.

(110) The minimal dependent sets of a matroid $M=(E, F)$, i.e., the minimal subsets of $E$ which are not members of $F$, are called the circuits of $M$.

(111) The circuits of $M(G)$ are the minimal non-empty sets $A \subseteq E$ such that $|A|=|u(A)|$.

(112) A set $J \subseteq E$ is a member of $F(G)$ iff $J$ together with the set $u(J)$ of nodes is a forest in $G$. 


\section{IX}

(113) Let $G$ be a directed graph. For any $R \subseteq V(G)$, a branching $B$ of $G$ rooted at $R$, is a forest of $G$ such that, for every $v \in V(G)$, there is a unique directed path in $B$ (possibly having zero edges) from some node in $R$ to $v$.

(114) The following problem is solved using matroid intersection

(115) Given any directed graph $G$, given a numerical weight $c_{j}$ for each $j \in E=$ $E(G)$, and given sets $R_{i} \subseteq V(G), i \in I$, find edge-disjoint branchings $B_{i}, i \in I$, rooted respectively at $R_{i}$, which minimize $s=\sum_{j} c_{j}, j \in \bigcup_{i \in I} B_{i}$.

(116) The problem easily reduces to the case where each $R_{i}$ consists of the same single node, $v_{0} \in V(G)$. That is, find $n=|I|$ edge-disjoint branchings $B_{i}$, each rooted at node $v_{0}$, which minimize $s$.

(117) Where $F(G)$ is as defined in (109), let $J \subseteq E$ be a member of $F_{1}$ iff it is the union of $n$ members of $F(G)$. By $(91), M_{1}=\left(E, F_{1}\right)$ is a matroid.

(118) Let $J \subseteq E$ be a member of $F_{2}$ iff no more than $n$ edges of $J$ are directed toward the same node in $V(G)$ and no edge of $J$ is directed toward $v_{0}$. Clearly, $M_{2}=\left(E, F_{2}\right)$ is a matroid:

(119) Theorem. A set $J \subseteq E$ is the edge-set of $n$ edge-disjoint branchings of $G$, rooted at node $v_{0} \in V(G)$, iff $|J|=n(|V(G)|-1)$ and $J \in F_{1} \cap F_{2}$.

This is a consequence of the following.

(120) Theorem. The maximum number of edge-disjoint branchings of $G$, rooted at $v_{0}$, equals the minimum over all $C, v_{0} \in C \subset V(G)$, of the number of edges having their tails in $C$ and their heads not in $C$.

(121) There is an algorithm for finding such a family of branchings in $G$, and in particular for partitioning a set $J$ as described in (119) into branchings as described in (119).

(122) Let $P_{1}$ and $P_{2}$ he the polyhedra of matroids $M_{1}$ and $M_{2}$ respectively. Let $H=\{x: x(E)=n(|V(G)|-1)\}$.

It follows from (45) that

(123) A vector $x \in \mathbb{R}_{E}$ is a vertex of $P_{1} \cap P_{2} \cap H$ iff it is the incidence vector of a set $J$ as described in (119).

(124) A variant of the matroid-intersection algorithm will find such an $x$ which minimizes $c \cdot x$. The case $n=1$ is treated in [4].

\section{$\mathrm{X}$}

(125) Let each $L_{i}$ be a commutative semigroup. We say $a \leq b$, for $\{a, b\} \subseteq L_{i}$, iff $a+d=b$ for some $d \in L_{i}$. 
(126) A function $f$ from $L_{0}$ into $L_{1}$ is called a $\psi$-function iff

(127) for every $\{a, d\} \subseteq L_{0}, f(a) \leq f(a+d)$; and

(128) for every $\{a, b, c\} \subseteq L_{0}, f(a+b+c)+f(c) \leq f(a+c)+f(b+c)$.

(129) $L_{i}$ is called a $\psi$-semigroup iff, for $\{a, b, c\} \subseteq L_{i}$,

$$
a+c+c=b+c+c \Longrightarrow a+c=b+c .
$$

For example, $L_{i}$ is a $\psi$-semigroup if it is cancellative or if it is idempotent.

(130) Theorem. If $f(\cdot)$ is a $\psi$-function from $L_{0}$ into $L_{1}, g(\cdot)$ is a $\psi$-function from $L_{1}$ into $L_{2}$, and $L_{1}$ is a $\psi$-semigroup, then $g(f(\cdot))$ is a $\psi$-function from $L_{0}$ into $L_{2}$.

(131) Theorem. A function $f$ from a lattice, $L_{0}$, into the non-negative reals, $L_{1}$, satisfies (128), where "+" in $L_{0}$ means " $\vee$ " and "+" in $L_{1}$ means ordinary addition, iff $f$ is non-decreasing, i.e., satisfies (127), and $f$ is submodular.

(132) Thus, $\beta$-functions can be obtained by composing $\psi$-functions.

(133) Theorem. A function $f$ from the non-negative reals into the nonnegative reals is a $\psi$-function, relative to addition in the image and preimage, iff it is non-decreasing and concave.

(134) Theorem. A function $f$ from a lattice, $L_{0}$, into a lattice, $L_{1}$, is a $\psi$ function, relative to joins " $\vee$ " in each, iff it is a join-homomorphism, i.e., for every $\{a, b\} \subseteq L_{0}, f(a \vee b)=f(a) \vee f(b)$.

Let $h(S)$ be any real (integer)-valued function of the elements $S \in L$ of a finite lattice $L$. In principle, an (integral) non-decreasing submodular function $f$ on $L$ can be obtained recursively from $h$ as follows:

(135) Theorem. For each $S \in L$, let $g(S)=\min [h(S), g(A)+g(B)-g(A \wedge B)]$ where $A<S, B<S$, and $A \vee B=S$. Then $g$ is submodular. For each $S \in L$, let $f(S)=\min g(A)$ where $S \leq A \in L$. Then $f$ is submodular and non-decreasing. If $h$ is submodular then $g=h$. If $h$ is submodular and non-decreasing then $f=h$.

(A similar construction was communicated to me by D. A. Higgs.)

(136) The $\beta$-functions on a finite lattice $L$ correspond to the members of a polyhedral cone $\beta(L)$ in the space of vectors $y=\left[y_{A}\right], A \in L-\{\emptyset\}$. Where $y_{\emptyset}=0, \beta(L)$ is the set of solutions to the system:

(137) $y_{A}+y_{B}-y_{A \vee B}-y_{A \wedge B} \geq 0$ and $y_{A \vee B}-y_{A} \geq 0$ for every $A \in L$ and $B \in L$.

(138) Characterizing the extreme rays of $\beta(L)$, in particular for $L=\{A: A \subseteq$ $E\}$, appears to be difficult. 


\section{References}

1. Dilworth, R.P., Dependence Relations in a Semimodular Lattice, Duke Math. J., 11 (1944), 575-587.

2. Edmonds, J. and Fulkerson, D.R., Transversals and Matroid Partition, J. Res. Nat. Bur. Standards, 69B (1965), 147-153.

3. Edmonds, J., Systems of Distinct Representatives and Linear Algebra, J. Res. Nat. Bur. Standards, 71B (1967), 241-245.

4. Edmonds, J., Optimum Branchings, J. Res. Nat. Bur. Standards, 71B (1967), 233240, reprinted with [5], 346-361.

5. Edmonds, J., Matroid Partition, Math. of the Decision Sciences, Amer. Math Soc. Lectures in Appl. Math., 11 (1968), 335-345.

6. Gale, D., Optimal assignments in an ordered set: an application of matroid theory, J. Combin. Theory, 4 (1968) 176-180.

7. Hoffman, A.J., Some Recent Applications of the Theory of Linear Inequalities to Extremal Combinatorial Analysis, Proc. Amer. Math. Soc. Symp. on Appl. Math., 10 (1960), 113-127.

8. Ingleton, A.W., A Note on Independence Functions and Rank, J. London Math. Soc., 34 (1959), 49-56.

9. Kruskal, J.B., On the shortest spanning subtree of a graph, Proc. Amer. Math. Soc., 7 (1956), 48-50.

10. Kuhn, H.W. and Tucker, A.W., eds., Linear inequalities and related systems, Annals of Math. Studies, no. 38, Princeton Univ. Press, 1956.

11. Lehman, A., A Solution of the Shannon Switching Game, J. Soc. Indust. Appl. Math., 12 (1964) 687-725.

12. Mirsky, L. and Perfect, H., Applications of the Notion of Independence to Problems in Combinatorial Analysis, J. Combin. Theory, 2 (1967), 327-357.

13. Nash-Williams, C.St.J.A., An application of matroids to graph theory, Proc. Int'l. Symposium on the Theory of Graphs, Rome 1966, Dunod.

14. Rado, R., A theorem on Independence Relations, Quart. J. Math., 13 (1942), 8389.

15. Rado, R., A Note on Independence Functions, Proc. London Math. Soc., 7 (1957), 300-320.

16. Tutte, W.T., Menger's Theorem for Matroids, J. Res. Nat. Bur. Standards, 69B (1965), 49-53. 